\title{
Coding for the Gaussian Interference Channel
}

\author{
Cibele Cristina Trinca, Jean-Claude Belfiore, Edson Donizete de Carvalho and Jozué Vieira Filho
}

\begin{abstract}
Interference is usually viewed as an obstacle to communication in wireless networks, so we developed a new methodology to quantize the channel coefficients in order to realize interference alignment onto a lattice. Our channel model is the same from the compute-and-forward strategy. In this work, we are going to explicit one example of channel quantization, which is related to the dimension 4 (real) or 2 (complex), and we will make use of the binary cyclotomic field $\mathbf{Q}\left(\xi_{8}\right)$, where $\xi_{8}$ is the 8-th root of unity.
\end{abstract}

Keywords - Channel coding, Lattice, Interference alignment.

\section{INTRODUCTION}

In a wireless network, a transmission from a single node is heard not only by the intended receiver, but also by all other nearby nodes. The resulting interference is usually viewed as highly undesirable and clever algorithms and protocols have been devised to avoid interference between transmitters.

In a recent work [1], Nazer and Gastpar proposed the compute-and-forward strategy as a physical-layer network coding scheme. They described a code structure based on nested lattices, whose algebraic structure makes the scheme reliable and efficient. The compute-and-forward strategy enables relays to decode linear equations of the transmitted messages using the noisy linear combinations provided by the channel. Each relay, indexed by $m=1,2, \ldots, M$, observes a noisy linear combination of the transmitted signals through the channel,

$$
y_{m}=\sum_{l=1}^{L} h_{m l} x_{l}+z_{m},
$$

where $h_{m l} \in \mathbf{C}$ are complex-valued channel coefficients, $x_{l} \in$ $\mathbf{C}^{n}$ such that $\left\|x_{l}\right\|^{2} \leq n P$ (in [1], Appendix $\mathrm{C}$, they argue that there exist fixed dithers that meet the power constraint) and $z_{m}$ is i.i.d. circularly symmetric complex Gaussian noise, $z_{m} \sim$ $\mathcal{C N}\left(\mathbf{0}, \mathbf{I}^{M \times M}\right)$. Let $h_{m}=\left[h_{m 1} \cdots h_{m L}\right]^{T}$ denote the vector of channel coefficients to relay $m$ and let $H=\left\{h_{m l}\right\}$ denote the entire channel matrix, where $T$ denotes the transpose. Note that by this convention the $m^{t h}$ row of $H$ is $h_{m}^{T}$.

However, in [1] we also have an equivalent channel induced by the modulo- $\Lambda$ transformation. In this "virtual" channel model each relay observes a $\mathbf{Z}[i]$-combination $\sum a_{m l} t_{l}$ of the lattice points corrupted by effective noise $z_{e q, m}$, that is,

$$
y_{m}=\sum_{l=1}^{L} a_{m l} t_{l}+z_{e q, m} .
$$

Cibele Cristina Trinca, Jean-Claude Belfiore, Edson Donizete de Carvalho and Jozué Vieira Filho Department of Electrical Engineering, FEISUNESP, Ilha Solteira-SP, Brazil; Department of Communications and Electronics, Télécom ParisTech, Paris, France; Department of Mathematics, FEISUNESP, Ilha Solteira-SP, Brazil; Department of Electrical Engineering, UNESP, São João da Boa Vista-SP, Brazil, E-mails: cibtrinca@yahoo.com.br, belfiore@enst.fr, edson@mat.feis.unesp.br, jozue.vieira@sjbv.unesp.br.
Transmitters send messages that take values in a primesized finite field and relays recover linear equations of the messages over the same field, thus we have and ideal physical layer interface for network coding. Even if the transmitters lack channel state information, this scheme can be applied.

The relaying strategy of the compute-and-forward is applicable to any configuration of sources, relays and destinations that are linked through linear channels with additive white Gaussian noise (AWGN). We refer to such configurations as AWGN networks.

\section{Problem Statement}

We developed a new methodology to quantize the channel coefficients in order to realize interference alignment onto a lattice. Our channel model is the same as the compute-andforward strategy, given by the equation 2 . So, in this work, we are going to explicit one example of channel quantization, this example is related to the dimensions 4 (real) or 2 (complex) and we will make use of the binary cyclotomic field $\mathbb{Q}\left(\xi_{8}\right)$, where $\xi_{8}$ is the 8 -th root of unity.

For this new methodology, we introduced an error criterion that measures, in a probabilistic sense, the error between the desired quantity and our estimate of it. Therefore, we have focused on choosing our estimate to minimize the expected or mean value of the square of the error, referred to as a minimum mean-square-error (MMSE) criterion.

But, in this work, we will only discuss about the methodology related to the channel approximation in order to realize interference alignment onto a lattice.

\section{PRELIMINARIES}

Lattices have been very useful in applications in communication theory and, in this work, we use lattices in order to realize interference alignment. In this section, we present basic concepts of the lattice theory.

Definition 3.1: Let $v_{1}, v_{2}, \ldots, v_{m}$ be a set of linearly independent vectors in $\mathbb{R}^{N}$ such that $m \leq N$. The set of the points

$$
\Lambda=\left\{x=\sum_{i=1}^{m} \lambda_{i} v_{i}, \text { where } \lambda_{i} \in \mathbb{Z}\right\}
$$

is called a lattice of rank $m$ and $\left\{v_{1}, v_{2}, \ldots, v_{m}\right\}$ is called a basis of the lattice.

So we have that a real lattice $\Lambda$ is simply a discrete set of vectors in real Euclidean $N$-space $\mathbf{R}^{N}$ that forms a group under ordinary vector addition, i.e., the sum or difference of any two vectors in $\Lambda$ is in $\Lambda$. Thus $\Lambda$ necessarily includes the all-zero $N$-tuple $\mathbf{0}$, and if $\lambda$ is in $\Lambda$, then so is its additive inverse $-\lambda$.

As an example, the set $\mathbf{Z}$ of all integers is the only onedimensional real lattice, up to scaling, and the prototype of 
all lattices. The set $\mathbf{Z}^{N}$ of all integers $N$-tuples is an $N$ dimensional real lattice, for any $N$, and its corresponding $\frac{N}{2}$ dimensional complex lattice is given by $\mathbf{Z}[i]^{\frac{N}{2}}$.

Lattices have only two principal structural characteristics. Algebraically, a lattice is a group; this property leads to the study of subgroups (sublattices) and partitions (coset decompositions) induced by such subgroups. Geometrically, a lattice is endowed with the properties of the space in which it is embedded, such as the Euclidean distance metric and the notion of volume in $\mathbf{R}^{N}$ [5].

\section{Quantization OF THE Channel Gains}

Suppose that our interference channel is complex-valued, specifically $a_{m l} \in\{\mathbf{Z}+i \mathbf{Z}\}$. We suppose that all lattices used by the legitimate user and the interferers are one of a certain lattice partition chain and extended by periodicity. Now the idea we want to develop is that the effect of a channel gain on a given user is to shift the lattice used by the user either to the left, if its channel gain is smaller than 1, or to the right, if it is larger than 1. It is very important that the channel gain does not remove the lattice from the initial chain of nested lattices.

\section{A. An example in dimension 4 (real) or 2 (complex)}

In this section, as we consider 2-dimensional complex valued vectors here, we will use the following lattice partition chain:

$$
\begin{gathered}
\frac{1}{1+i} \mathbf{Z}[i]^{2} \supset \frac{1}{1+i} D_{4} \supset \mathbf{Z}[i]^{2} \supset D_{4} \supset(1+i) \mathbf{Z}[i]^{2} \supset \\
\supset(1+i) D_{4} \supset 2 \mathbf{Z}[i]^{2} .
\end{gathered}
$$

So each transmitter is using one of the lattices of this chain (which is extended by periodicity so that it is a doubly infinite chain). Now we write, for a given user, how its codeword can be transformed so that we can perform the channel quantization. We will make use of the binary cyclotomic field $\mathbb{Q}\left(\xi_{8}\right)$.

We will consider the following Galois extensions:

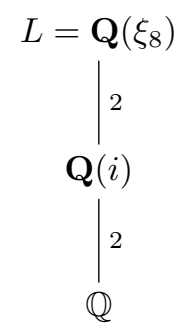

We have that the Galois groups of $\left[\mathbf{Q}\left(\xi_{8}\right): \mathbf{Q}(i)\right]$ and $[\mathbf{Q}(i): \mathbf{Q}]$ are given by $\operatorname{Gal}\left(\mathbf{Q}\left(\xi_{8}\right) / \mathbf{Q}(i)\right)=\left\{\sigma_{1}=i d\right.$ : $\mathbf{Q}\left(\xi_{8}\right) \rightarrow \mathbf{Q}\left(\xi_{8}\right)$ and $\left.\sigma_{2}: \mathbf{Q}\left(\xi_{8}\right) \rightarrow \mathbf{Q}\left(\xi_{8}\right) ; \sigma_{2}\left(\xi_{8}\right)=-\xi_{8}\right\}$ and $\operatorname{Gal}(\mathbf{Q}(i) / \mathbf{Q})=\left\{\sigma_{1}=i d: \mathbf{Q}(i) \rightarrow \mathbf{Q}(i)\right.$ and $\sigma_{2}:$ $\left.\mathbf{Q}(i) \rightarrow \mathbf{Q}(i) ; \sigma_{2}(i)=-i\right\}$, respectively, where $i d$ is the identity map.

Since $\left\{1, \xi_{8}\right\}$ is a $\mathbf{Z}[i]$-basis of $O_{L}$, so the matrix

$$
M_{0}=\left(\begin{array}{cc}
\sigma_{1}(1) & \sigma_{2}(1) \\
\sigma_{1}\left(\xi_{8}\right) & \sigma_{2}\left(\xi_{8}\right)
\end{array}\right)=\left(\begin{array}{cc}
1 & 1 \\
\xi_{8} & -\xi_{8}
\end{array}\right)
$$

is the generator matrix of the complex algebraic lattice $\sigma\left(O_{L}\right)$ ([4], chapter 2).

If we take $M_{0}^{\prime}=\frac{1}{\sqrt{2}} M_{0}$, we have that $M_{0}^{\prime}$ and $U=\frac{1}{\sqrt{2}} M_{0}^{T}$ are unitary matrices. Therefore, $\sigma\left(O_{L}\right)$ is isomorphic to the $\mathbf{Z}[i]^{2}$-lattice.

At the receiver, we suppose that we apply $U$ to the received vector of 2 to get

$$
\bar{y}_{m}=U y_{m}=\sum_{l=1}^{L} a_{m l} U t_{l}+U z_{e q, m} .
$$

Now let's take a look at the vectors of the form $a_{m l} U t_{l}$. For sake of simplicity of notations, we denote it by

$$
\bar{x}=h \cdot U \cdot x,
$$

where $x=t_{l}$ is the lattice point transmitted by the considered user and $h=a_{m l}$ is the channel coefficient. We can rewrite it now as

$$
\left(\begin{array}{cc}
h & 0 \\
0 & h
\end{array}\right) \cdot U \cdot x=H \cdot U \cdot x .
$$

The idea we want to develop is to quantize the diagonal matrix $H$ by the diagonal matrix whose elements are components of the canonical embedding of the power (positive or negative) of an element of $O_{L}$ with absolute algebraic norm [4] equal to 2 .

We have that 2 is totally ramified [4] in $\mathbf{Q}\left(\xi_{8}\right)$ and $2 \mathbf{Z}\left[\xi_{8}\right]=$ $(2)=\Im^{4}$, where $\Im=\left(1+\xi_{8}\right)$. Then $\Im$ is the ideal in $O_{L}=$ $\mathbf{Z}\left[\xi_{8}\right]$ generated by $\mu=1+\xi_{8}$ and $\Im^{k}, k \in \mathbf{Z}$, is an ideal of $O_{L}$ generated by $\mu^{k}$.

Now we will approximate the matrix $H$ with the canonical embedding of the generator $\mu^{k}$ of $\Im^{k}, k \in \mathbf{Z}$, and we are going to make use of the following proposition:

Proposition 4.1: We have that $\left\{u_{k}, u_{k} \xi_{8}\right\}$ is a $\mathbf{Z}[i]$-basis of $u_{k} \mathbf{Z}\left[\xi_{8}\right]=u_{k} O_{L}$, where $\left\{1, \xi_{8}\right\}$ is a $\mathbf{Z}[i]$-basis of $O_{L}$ [4] and $u_{k}=\mu^{k}$ is the generator of the ideal $\Im^{k}$, with $k \in \mathbb{Z}$.

Proof: Let $u_{k}=\mu^{k}$ be the generator of the ideal $\Im^{k}$, where $k \in \mathbf{Z}$ and $\mu=1+\xi_{8}$. Let $x \in u_{k} O_{L}$, then $x=u_{k} \alpha$, with $\alpha \in O_{L}$. So

$$
\begin{aligned}
& x=u_{k}\left(a+b \xi_{8}\right), \text { where } a, b \in \mathbf{Z}[i], \text { if, and only if, } \\
& x=u_{k} a+u_{k} b \xi_{8}=a u_{k}+b\left(u_{k} \xi_{8}\right), \text { with } a, b \in \mathbf{Z}[i],
\end{aligned}
$$

therefore $\left\{u_{k}, u_{k} \xi_{8}\right\}$ generates $u_{k} O_{L}$. We will prove now that $\left\{u_{k}, u_{k} \xi_{8}\right\}$ is linearly independent. In fact, let $a, b \in \mathbf{Z}[i]$, then

$$
\begin{gathered}
a u_{k}+b u_{k} \xi_{8}=0 \Leftrightarrow a u_{k} u_{k}^{-1}+b u_{k} u_{k}^{-1} \xi_{8}=0 \Leftrightarrow \\
\Leftrightarrow a+b \xi_{8}=0 \Leftrightarrow a=b=0 .
\end{gathered}
$$

So $\left\{u_{k}, u_{k} \xi_{8}\right\}$ is a $\mathbf{Z}[i]$-basis of $u_{k} O_{L}$.

Then, by the proposition 4.1 , we have that the generator matrix of the complex algebraic lattice $\sigma\left(u_{k} O_{L}\right)$ [4] is given by (note that the transposed matrix has the same properties)

$$
M_{k}=\left(\begin{array}{cc}
u_{k} & 0 \\
0 & \sigma\left(u_{k}\right)
\end{array}\right) \cdot\left(\begin{array}{cc}
1 & \xi_{8} \\
1 & -\xi_{8}
\end{array}\right),
$$

so the matrix $H$ can be approximated by

$$
M_{k}^{\prime}=\left(\begin{array}{cc}
u_{k} & 0 \\
0 & \sigma\left(u_{k}\right)
\end{array}\right) \text {. }
$$


The following section will show us that the canonical embeddings of $\Im=\mu O_{L}(k=1), \Im^{2}=\mu^{2} O_{L}(k=2)$, $\Im^{3}=\mu^{3} O_{L}(k=3)$ and $\Im^{4}=\mu^{4} O_{L}(k=4)$ are simply the lattices $D_{4}, R \mathbf{Z}^{4}, R D_{4}$ and $2 \mathbf{Z}^{4}$, respectively, where $R$ is the rotation transformation.

1) Interference alignment onto a lattice for $k \geq 0$ : We will see, in this section, that the quantization of the channel coefficients will give us the lattices $D_{4}, R \mathbf{Z}^{4}, R D_{4}$ and $2 \mathbf{Z}^{4}$, when $k=1,2,3$ and 4 , respectively. So we will have a lattice partition chain and such lattices are related to $k=1,2,3$ and 4. We will also obtain the extension by periodicity of this lattice partition chain.

In fact, for $k=1$, let

$$
\left(\begin{array}{cc}
\mu & 0 \\
0 & \sigma(\mu)
\end{array}\right) \cdot\left(\begin{array}{cc}
1 & \xi_{8} \\
1 & -\xi_{8}
\end{array}\right)=\left(\begin{array}{cc}
1 & \xi_{8} \\
1 & -\xi_{8}
\end{array}\right) \cdot M_{\mu}
$$

It is easy to see that $M_{\mu}$ is a generator matrix of the lattice $D_{4}$ seen as a $\mathbf{Z}[i]$-lattice; in fact, the matrix $M_{\mu}$ with real entries is given by

$$
\left(\begin{array}{cccc}
1 & 0 & 0 & -1 \\
0 & 1 & 1 & 0 \\
1 & 0 & 1 & 0 \\
0 & 1 & 0 & 1
\end{array}\right)
$$

and we can note that in each column of $M_{\mu}$ the sum of the coefficients is even, therefore the lattice $\Lambda_{1}$ generated by $M_{\mu}$ is a sublattice of $D_{4}$. Moreover, we have that $\operatorname{Vol}\left(\Lambda_{1}\right)=2=$ $\operatorname{Det}\left(M_{\mu}\right)=\operatorname{Vol}\left(D_{4}\right)$, then the index $\left|D_{4} / \Lambda_{1}\right|$ is equal to 1 and so $\Lambda_{1}=D_{4}$.

With these properties, we have that $M_{\mu}$ is a generator matrix of $D_{4}$ seen as a $\mathbf{Z}[i]$-lattice.

This means that, if $u_{1}=\mu$ generates the ideal $\mu O_{L}$, then the matrix $M_{\mu}$ is a generator matrix of the lattice $D_{4}$ in 4 , whose position compared to $\mathbf{Z}[i]^{2}$ is equal to $k=1$. We have that $\mathbf{Z}[i]^{2}$ has position 0 (note that $M_{0}^{T}$ is a generator matrix for the lattice $\mathbf{Z}[i]^{2}$ ).

Now, for $k=2$, let

$$
\left(\begin{array}{cc}
\mu^{2} & 0 \\
0 & \sigma(\mu)^{2}
\end{array}\right) \cdot\left(\begin{array}{cc}
1 & \xi_{8} \\
1 & -\xi_{8}
\end{array}\right)=\left(\begin{array}{cc}
1 & \xi_{8} \\
1 & -\xi_{8}
\end{array}\right) \cdot M_{\mu^{2}}
$$

Observe that $M_{\mu^{2}}=\left(M_{\mu}\right)^{2}$ and $\left(M_{\mu}\right)^{2}$ with real entries is given by

$$
\left(M_{\mu}\right)^{2}=\left(\begin{array}{cccc}
1 & -1 & 0 & -2 \\
1 & 1 & 2 & 0 \\
2 & 0 & 1 & -1 \\
0 & 2 & 1 & 1
\end{array}\right)
$$

By straightforward computation, it follows that $\left(M_{\mu}\right)^{2}=$ $R \cdot M$, where $R$ is the rotation transformation given by

$$
\begin{gathered}
R=\left(\begin{array}{cccc}
1 & 1 & 0 & 0 \\
1 & -1 & 0 & 0 \\
0 & 0 & 1 & 1 \\
0 & 0 & 1 & -1
\end{array}\right) \text { and } \\
M=\left(\begin{array}{cccc}
1 & 0 & 1 & -1 \\
0 & -1 & -1 & -1 \\
1 & 1 & 1 & 0 \\
1 & -1 & 0 & -1
\end{array}\right) .
\end{gathered}
$$

So we have that $\left(M_{\mu}\right)^{2}=R \cdot M$ is a generator matrix of the lattice $R \mathbf{Z}^{4} \simeq(1+i) \mathbf{Z}[i]^{2}$ seen as a $\mathbf{Z}[i]$-lattice, since the matrix $M$ is unimodular.
This means that, if $u_{2}=\mu^{2}$ generates the ideal $\mu^{2} O_{L}$, then the matrix $M_{\mu^{2}}=\left(M_{\mu}\right)^{2}$ is a generator matrix of the lattice $(1+i) \mathbf{Z}[i]^{2}$ in 4 , whose position compared to $\mathbf{Z}[i]^{2}$ is equal to $k=2$.

For $k=3$, let

$$
\left(\begin{array}{cc}
\mu^{3} & 0 \\
0 & \sigma(\mu)^{3}
\end{array}\right) \cdot\left(\begin{array}{cc}
1 & \xi_{8} \\
1 & -\xi_{8}
\end{array}\right)=\left(\begin{array}{cc}
1 & \xi_{8} \\
1 & -\xi_{8}
\end{array}\right) \cdot M_{\mu^{3}} .
$$

Observe that $M_{\mu^{3}}=\left(M_{\mu}\right)^{3}$ and the matrix $\left(M_{\mu}\right)^{3}$ with real entries is given by

$$
\left(M_{\mu}\right)^{3}=\left(\begin{array}{cccc}
1 & -3 & -1 & -3 \\
3 & 1 & 3 & -1 \\
3 & -1 & 1 & -3 \\
1 & 3 & 3 & 1
\end{array}\right)
$$

By straightforward computation, it follows that $\left(M_{\mu}\right)^{3}=$ $R \cdot N$, where $R$ is the rotation transformation given by

$$
\begin{aligned}
R & =\left(\begin{array}{cccc}
1 & 1 & 0 & 0 \\
1 & -1 & 0 & 0 \\
0 & 0 & 1 & 1 \\
0 & 0 & 1 & -1
\end{array}\right) \text { and } \\
N & =\left(\begin{array}{cccc}
2 & -1 & 1 & -2 \\
-1 & -2 & -2 & -1 \\
2 & 1 & 2 & -1 \\
1 & -2 & -1 & -2
\end{array}\right) .
\end{aligned}
$$

Note that in each column of $N$ the sum of the coefficients is even, therefore the lattice $\Lambda_{2}$ generated by $N$ is a sublattice of $D_{4}$. Moreover, we have that $\operatorname{Vol}\left(\Lambda_{2}\right)=2=\operatorname{Det}(N)=$ $\operatorname{Vol}\left(D_{4}\right)$, then the index $\left|D_{4} / \Lambda_{2}\right|$ is equal to 1 and so $\Lambda_{2}=$ $D_{4}$.

With these properties we have that $\left(M_{\mu}\right)^{3}=R \cdot N$ is a generator matrix of $R D_{4} \simeq(1+i) D_{4}$ seen as a $\mathbf{Z}[i]$-lattice.

This means that, if $u_{3}=\mu^{3}$ generates the ideal $\mu^{3} O_{L}$, then the matrix $M_{\mu^{3}}=\left(M_{\mu}\right)^{3}$ is a generator matrix of the lattice $(1+i) D_{4}$ in 4 , whose position compared to $\mathbf{Z}[i]^{2}$ is equal to $k=3$.

And, for $k=4$, let

$$
\left(\begin{array}{cc}
\mu^{4} & 0 \\
0 & \sigma(\mu)^{4}
\end{array}\right) \cdot\left(\begin{array}{cc}
1 & \xi_{8} \\
1 & -\xi_{8}
\end{array}\right)=\left(\begin{array}{cc}
1 & \xi_{8} \\
1 & -\xi_{8}
\end{array}\right) \cdot M_{\mu^{4}} .
$$

Observe that $M_{\mu^{4}}=\left(M_{\mu}\right)^{4}$ and the matrix $\left(M_{\mu}\right)^{4}$ with real entries is given by

$$
\left(M_{\mu}\right)^{4}=2\left(\begin{array}{cccc}
0 & -3 & -2 & -2 \\
3 & 0 & 2 & -2 \\
2 & -2 & 0 & -3 \\
2 & 2 & 3 & 0
\end{array}\right)=2 P .
$$

So we have that $\left(M_{\mu}\right)^{4}=2 \cdot P$ is a generator matrix of the lattice $2 \mathbf{Z}^{4} \simeq 2 \mathbf{Z}[i]^{2}$ seen as a $\mathbf{Z}[i]$-lattice, since $P$ is unimodular.

This means that, if $u_{4}=\mu^{4}$ generates the ideal $\mu^{4} O_{L}$, then the matrix $M_{\mu^{4}}=\left(M_{\mu}\right)^{4}$ is a generator matrix of the lattice $2 \mathbf{Z}[i]^{2}$ in 4 , whose position compared to $\mathbf{Z}[i]^{2}$ is equal to $k=4$.

Now the two following propositions will give us the extension by periodicity of the lattice partition chain in 4 for the positive positions, that is, $k \geq 0$ :

Proposition 4.2: For $k=2 \alpha, \alpha \in \mathbf{N}^{*}$, we have that $\left(M_{\mu}\right)^{k=2 \alpha}$ is a generator matrix of the lattice $R^{\alpha} \mathbf{Z}^{4} \simeq(1+$ $i)^{\alpha} \mathbf{Z}[i]^{2}$ seen as a $\mathbf{Z}[i]$-lattice. 
Proof: By previously, for $k=2$ and 4, we have the matrices $M_{\mu^{2}}$ and $M_{\mu^{4}}$ as being generator matrices of the lattices $R \mathbf{Z}^{4} \simeq(1+i) \mathbf{Z}[i]^{2}$ and $2 \mathbf{Z}^{4} \simeq 2 \mathbf{Z}[i]^{2}$, respectively.

By straightforward computation, we can see that the matrix $M_{\mu^{2}}=\left(M_{\mu}\right)^{2}$ is equivalent to the rotation matrix $R$. Now, for $k \geq 1$, we can show by induction over $\alpha$ that for $k=2 \alpha$, $\alpha \in \mathbf{N}^{*}$, if $u_{2 \alpha}=\mu^{2 \alpha}$ generates the ideal $\mu^{2 \alpha} O_{L}$, then the matrix $\left(M_{\mu}\right)^{2 \alpha}$ is a generator matrix of the lattice $R^{\alpha} \mathbf{Z}^{4} \simeq$ $(1+i)^{\alpha} \mathbf{Z}[i]^{2}$.

But if $\alpha$ is even, we have $\alpha=2 \beta$, where $\beta \in \mathbf{N}^{*}$, then $R^{\alpha} \mathbf{Z}^{4}=2^{\alpha / 2} \mathbf{Z}^{4} \simeq 2^{\alpha / 2} \mathbf{Z}[i]^{2}$.

Now, if $\alpha$ is odd, we have $\alpha=2 \beta+1$, where $\beta \in \mathbf{N}$, then $R^{\alpha} \mathbf{Z}^{4}=2^{(\alpha-1) / 2} R \mathbf{Z}^{4} \simeq 2^{(\alpha-1) / 2}(1+i) \mathbf{Z}[i]^{2}$.

Proposition 4.3: For $k=2 \alpha+1, \alpha \in \mathbf{N}^{*}$, we have that $\left(M_{\mu}\right)^{k=2 \alpha+1}$ is a generator matrix of the lattice $R^{\frac{k-1}{2}} D_{4}=$ $R^{\alpha} D_{4} \simeq(1+i)^{\alpha} D_{4}$ seen as a $\mathbf{Z}[i]$-lattice.

Proof: The proof is analogous to the previous proposition. So if $\alpha$ is even, we have $\alpha=2 \beta$, where $\beta \in \mathbf{N}^{*}$, then $R^{\alpha} D_{4}=2^{\alpha / 2} D_{4}$.

Now, if $\alpha$ is odd, we have $\alpha=2 \beta+1$, where $\beta \in \mathbf{N}$, then $R^{\alpha} D_{4}=2^{(\alpha-1) / 2} R D_{4} \simeq 2^{(\alpha-1) / 2}(1+i) D_{4}$.

Thus, in this section, we have the interference alignment onto a lattice for $k \geq 0$. Now, in the following section, we will show that the canonical embeddings of $\Im^{-1}=\mu^{-1} O_{L}$ $(k=-1), \Im^{-2}=\mu^{-2} O_{L}(k=-2), \Im^{-3}=\mu^{-3} O_{L}(k=$ $-3)$ and $\Im^{-4}=\mu^{-4} O_{L}(k=-4)$ are simply the lattices $R^{-1} D_{4}, R^{-1} \mathbf{Z}^{4}, \frac{1}{2} D_{4}$ and $\frac{1}{2} \mathbf{Z}^{4}$, respectively, where $R$ is the rotation transformation and $R^{-1}$ is its inverse.

2) Interference alignment onto a lattice for $k<0$ : We will see, in this section, that the quantization of the channel coefficients will give us the lattices $R^{-1} D_{4}, R^{-1} \mathbf{Z}^{4}, \frac{1}{2} D_{4}$ and $\frac{1}{2} \mathbf{Z}^{4}$, when $k=-1,-2,-3$ and -4 , respectively. Thus, we will have a lattice partition chain and such lattices are related to $k=-1,-2,-3$ and -4 . We will also obtain the extension by periodicity of this lattice partition chain.

In fact, for $k=-1$, let

$$
\begin{gathered}
\left(\begin{array}{cc}
\mu^{-1} & 0 \\
0 & \sigma(\mu)^{-1}
\end{array}\right) \cdot\left(\begin{array}{cc}
1 & \xi_{8} \\
1 & -\xi_{8}
\end{array}\right)= \\
=\left(\begin{array}{cc}
1 & \xi_{8} \\
1 & -\xi_{8}
\end{array}\right) \cdot M_{\mu^{-1}}
\end{gathered}
$$

Let $M$ be the matrix such that $M_{\mu^{-1}}=\frac{1}{(1+i)} M$, that is,

$$
M=\left(\begin{array}{cc}
i & 1 \\
-i & i
\end{array}\right)
$$

We have that $M$ is a generator matrix of the lattice $D_{4}$ seen as a $\mathbf{Z}[i]$-lattice; in fact, the matrix $M$ with real entries is given by

$$
\left(\begin{array}{cccc}
0 & -1 & 1 & 0 \\
1 & 0 & 0 & 1 \\
0 & 1 & 0 & -1 \\
-1 & 0 & 1 & 0
\end{array}\right)
$$

and we note that in each column of $M$ the sum of the coefficients is even, therefore the lattice $\Lambda_{-1}$ generated by $M$ is a sublattice of $D_{4}$. Moreover, we have that $\operatorname{Vol}\left(\Lambda_{-1}\right)=$ $2=\operatorname{Det}(M)=\operatorname{Vol}\left(D_{4}\right)$, then the index $\left|D_{4} / \Lambda_{-1}\right|$ is equal to 1 and so $\Lambda_{-1}=D_{4}$.
With these properties we have that $M$ is a generator matrix of $D_{4}$ seen as a $\mathbf{Z}[i]$-lattice. So $M_{\mu^{-1}}$ is a generator matrix of the lattice $\frac{1}{(1+i)} D_{4}$ seen as a $\mathbf{Z}[i]$-lattice.

This means that, if $u_{-1}=\mu^{-1}$ generates the ideal $\mu^{-1} O_{L}$, then the matrix $M_{\mu^{-1}}$ is a generator matrix of the lattice $\frac{1}{(1+i)} D_{4}$ in 4 , whose position compared to $\mathbf{Z}[i]^{2}$ is equal to $k=-1$ (left side).

Now, for $k=-2$, let

$$
\begin{gathered}
\left(\begin{array}{cc}
\mu^{-2} & 0 \\
0 & \sigma(\mu)^{-2}
\end{array}\right) \cdot\left(\begin{array}{cc}
1 & \xi_{8} \\
1 & -\xi_{8}
\end{array}\right)= \\
=\left(\begin{array}{cc}
1 & \xi_{8} \\
1 & -\xi_{8}
\end{array}\right) \cdot M_{\mu^{-2}} .
\end{gathered}
$$

So $M_{\mu^{-2}}=\left(M_{\mu^{-1}}\right)^{2}=\frac{1}{(1+i)} M$, where $M$ is given by

$$
M=\left(\begin{array}{cc}
-1 & 1+i \\
1-i & -1
\end{array}\right)
$$

The matrix $M$ with real entries is given by

$$
M=\left(\begin{array}{cccc}
-1 & 0 & 1 & -1 \\
0 & -1 & 1 & 1 \\
1 & 1 & -1 & 0 \\
-1 & 1 & 0 & -1
\end{array}\right)
$$

Thus, we have that $\left(M_{\mu^{-1}}\right)^{2}=\frac{1}{(1+i)} M$ is a generator matrix of the lattice $R^{-1} \mathbf{Z}^{4} \simeq \frac{1}{(1+i)} \mathbf{Z}[i]^{2}$ seen as a $\mathbf{Z}[i]$-lattice, since $M$ is unimodular.

This means that, if $u_{-2}=\mu^{-2}$ generates the ideal $\mu^{-2} O_{L}$, then the matrix $M_{\mu^{-2}}=\left(M_{\mu^{-1}}\right)^{2}$ is a generator matrix of the lattice $\frac{1}{(1+i)} \mathbf{Z}[i]^{2}$ in 4 , whose position compared to $\mathbf{Z}[i]^{2}$ is equal to $k=-2$.

For $k=-3$, let

$$
\begin{gathered}
\left(\begin{array}{cc}
\mu^{-3} & 0 \\
0 & \sigma(\mu)^{-3}
\end{array}\right) \cdot\left(\begin{array}{cc}
1 & \xi_{8} \\
1 & -\xi_{8}
\end{array}\right)= \\
=\left(\begin{array}{cc}
1 & \xi_{8} \\
1 & -\xi_{8}
\end{array}\right) \cdot M_{\mu^{-3}}
\end{gathered}
$$
by

Observe that $M_{\mu^{-3}}=\left(M_{\mu^{-1}}\right)^{3}=\frac{1}{2} M$, where $M$ is given

$$
M=\left(\begin{array}{cc}
-2-i & 1+2 i \\
2-i & -2-i
\end{array}\right)
$$

The matrix $M$ with real entries is given by

$$
M=\left(\begin{array}{cccc}
-2 & 1 & 1 & -2 \\
-1 & -2 & 2 & 1 \\
2 & 1 & -2 & 1 \\
-1 & 2 & -1 & -2
\end{array}\right) .
$$

Note that in each column of $M$ the sum of the coefficients is even, therefore the lattice $\Lambda_{-3}$ generated by $M$ is a sublattice of $D_{4}$. Moreover, we have that $\operatorname{Vol}\left(\Lambda_{-3}\right)=2=\operatorname{Det}(M)=$ $\operatorname{Vol}\left(D_{4}\right)$, then the index $\left|D_{4} / \Lambda_{-3}\right|$ is equal to 1 and so $\Lambda_{-3}=$ $D_{4}$.

With these properties we have that $\left(M_{\mu^{-1}}\right)^{3}=\frac{1}{2} M$ is a generator matrix of $\frac{1}{2} D_{4}$ seen as a $\mathbf{Z}[i]$-lattice.

This means that, if $u_{-3}=\mu^{-3}$ generates the ideal $\mu^{-3} O_{L}$, then the matrix $M_{\mu^{-3}}=\left(M_{\mu^{-1}}\right)^{3}$ is a generator matrix of the lattice $\frac{1}{2} D_{4}$ in 4 , whose position compared to $\mathbf{Z}[i]^{2}$ is equal to $k=-3$. 
And, for $k=-4$, let

$$
\begin{gathered}
\left(\begin{array}{cc}
\mu^{-4} & 0 \\
0 & \sigma(\mu)^{-4}
\end{array}\right) \cdot\left(\begin{array}{cc}
1 & \xi_{8} \\
1 & -\xi_{8}
\end{array}\right)= \\
=\left(\begin{array}{cc}
1 & \xi_{8} \\
1 & -\xi_{8}
\end{array}\right) \cdot M_{\mu^{-4}}
\end{gathered}
$$
by

Observe that $M_{\mu^{-4}}=\left(M_{\mu^{-1}}\right)^{4}=\frac{1}{2} M$, where $M$ is given

$$
M=\left(\begin{array}{cc}
-3 i & -2+2 i \\
2+2 i & -3 i
\end{array}\right)
$$

The matrix $M$ with real entries is given by

$$
M=\left(\begin{array}{cccc}
0 & 3 & -2 & -2 \\
-3 & 0 & 2 & -2 \\
2 & -2 & 0 & 3 \\
2 & 2 & -3 & 0
\end{array}\right)
$$

Thus, we have that $\left(M_{\mu^{-1}}\right)^{4}=\frac{1}{2} M$ is a generator matrix of the lattice $\frac{1}{2} \mathbf{Z}^{4} \simeq \frac{1}{2} \mathbf{Z}[i]^{2}$ seen as a $\mathbf{Z}[i]$-lattice, since $M$ is unimodular.

This means that, if $u_{-4}=\mu^{-4}$ generates the ideal $\mu^{-4} O_{L}$, then the matrix $M_{\mu^{-4}}=\left(M_{\mu^{-1}}\right)^{4}$ is a generator matrix of the lattice $\frac{1}{2} \mathbf{Z}[i]^{2}$ in 4 , whose position compared to $\mathbf{Z}[i]^{2}$ is equal to $k=-4$.

Now the two following propositions will give us the extension by periodicity of the lattice partition chain in 4 for the negative positions, that is, $k \leq-1$ :

Proposition 4.4: For $k=-2 \alpha, \alpha \in \mathbf{N}^{*}$, we have that $\left(M_{\mu^{-1}}\right)^{k=2 \alpha}$ is a generator matrix of the lattice $R^{-\alpha} \mathbf{Z}^{4} \simeq$ $\left(\frac{1}{(1+i)}\right)^{\alpha} \mathbf{Z}[i]^{2}$ seen as a $\mathbf{Z}[i]$-lattice.

Proof: By previously, for $k=-2$ and -4 , we have the matrices $M_{\mu^{-2}}$ and $M_{\mu^{-4}}$ as being generator matrices of the lattices $R^{-1} \mathbf{Z}^{4} \simeq \frac{1}{(1+i)} \mathbf{Z}[i]^{2}$ and $\frac{1}{2} \mathbf{Z}^{4} \simeq \frac{1}{2} \mathbf{Z}[i]^{2}$, respectively.

By straightforward computation we can see that the matrix $M_{\mu^{-2}}=\left(M_{\mu^{-1}}\right)^{2}=\frac{1}{2} M$, where

$$
M=\left(\begin{array}{cccc}
-1 & -1 & 2 & 0 \\
1 & -1 & 0 & 2 \\
0 & 2 & -1 & -1 \\
-2 & 0 & 1 & -1
\end{array}\right)
$$

is equivalent (equivalent matrices generate the same lattice) to the matrix $R^{-1}=\frac{1}{2} R$ (inverse of the matrix $R$ ), where

$$
R=\left(\begin{array}{cccc}
1 & 1 & 0 & 0 \\
1 & -1 & 0 & 0 \\
0 & 0 & 1 & 1 \\
0 & 0 & 1 & -1
\end{array}\right)
$$

Now, for $k \leq-1$, we can see by induction that

$$
\begin{gathered}
\left(\begin{array}{cc}
\mu^{k} & 0 \\
0 & \sigma(\mu)^{k}
\end{array}\right) \cdot\left(\begin{array}{cc}
1 & \xi_{8} \\
1 & -\xi_{8}
\end{array}\right)= \\
\left(\begin{array}{cc}
1 & \xi_{8} \\
1 & -\xi_{8}
\end{array}\right) \cdot\left(M_{\mu^{-1}}\right)^{-k},
\end{gathered}
$$

where $\left(M_{\mu^{-1}}\right)^{-k}=M_{\mu^{k}}$.

Let's suppose, by induction hypothesis, that $\left(M_{\mu^{-1}}\right)^{2 \alpha}, \alpha \in$ $\mathbf{N}^{*}$, is a generator matrix of the lattice $R^{-\alpha} \mathbf{Z}^{4}$.
We will show, for $k=-2 \alpha-2, \alpha \in \mathbf{N}^{*}$, that the lattice $R^{-\alpha-1} \mathbf{Z}^{4}$ has a generator matrix as being the matrix $\left(M_{\mu^{-1}}\right)^{2 \alpha+2}$. In fact, we have

$$
\begin{gathered}
\left(\begin{array}{cc}
\mu^{-2 \alpha-2} & 0 \\
0 & \sigma(\mu)^{-2 \alpha-2}
\end{array}\right) \cdot\left(\begin{array}{cc}
1 & \xi_{8} \\
1 & -\xi_{8}
\end{array}\right)= \\
=\left(\begin{array}{cc}
1 & \xi_{8} \\
1 & -\xi_{8}
\end{array}\right) \cdot\left(M_{\mu^{-1}}\right)^{2 \alpha+2},
\end{gathered}
$$

where $\left(M_{\mu^{-1}}\right)^{2 \alpha+2}=M_{\mu^{-2 \alpha-2}}$.

Then $\left(M_{\mu^{-1}}\right)^{2 \alpha+2}=\left(\left(M_{\mu^{-1}}\right)^{2}\right)\left(\left(M_{\mu^{-1}}\right)^{2 \alpha}\right)$, using the induction hypothesis and the fact that $R^{-1}$ is equivalent to $\left(M_{\mu^{-1}}\right)^{2}$, we have $\left(M_{\mu^{-1}}\right)^{2 \alpha+2}$ as a generator matrix of the lattice $R^{-\alpha-1} \mathbf{Z}^{4} \simeq\left(\frac{1}{(1+i)}\right)^{\alpha+1} \mathbf{Z}[i]^{2}$.

Therefore we showed that for $k=-2 \alpha, \alpha \in \mathbf{N}^{*}$, if $u_{-2 \alpha}=$ $\mu^{-2 \alpha}$ generates the ideal $\mu^{-2 \alpha} O_{L}$, then the matrix $\left(M_{\mu^{-1}}\right)^{2 \alpha}$ is a generator matrix of the lattice $R^{-\alpha} \mathbf{Z}^{4} \simeq\left(\frac{1}{(1+i)}\right)^{\alpha} \mathbf{Z}[i]^{2}$.

But if $\alpha$ is even, we have $\alpha=2 \beta$, where $\beta \in \mathbf{N}^{*}$, then $R^{-\alpha} \mathbf{Z}^{4}=\left(\frac{1}{2}\right)^{\alpha / 2} \mathbf{Z}^{4} \simeq\left(\frac{1}{2}\right)^{\alpha / 2} \mathbf{Z}[i]^{2}$.

Now, if $\alpha$ is odd, we have $\alpha=2 \beta+1$, where $\beta \in \mathbf{N}$, then $R^{-\alpha} \mathbf{Z}^{4}=\left(\frac{1}{2}\right)^{(\alpha-1) / 2} R^{-1} \mathbf{Z}^{4} \simeq\left(\frac{1}{2}\right)^{(\alpha-1) / 2}\left(\frac{1}{1+i}\right) \mathbf{Z}[i]^{2}$.

Proposition 4.5: For $k=-2 \alpha+1, \alpha \in \mathbf{N}^{*}$, we have that $\left(M_{\mu^{-1}}\right)^{-k=2 \alpha-1}$ is a generator matrix of the lattice $R^{-\alpha=(k-1) / 2} D_{4} \simeq\left(\frac{1}{1+i}\right)^{\alpha} D_{4}$ seen as a $\mathbf{Z}[i]$-lattice.

Proof: The proof is analogous to the previous proposition. So if $\alpha$ is even, we have $\alpha=2 \beta$, where $\beta \in \mathbf{N}^{*}$, then $R^{-\alpha} D_{4}=\left(\frac{1}{2}\right)^{\alpha / 2} D_{4}$.

Now, if $\alpha$ is odd, we have $\alpha=2 \beta+1$, where $\beta \in \mathbf{N}$, then $R^{-\alpha} D_{4}=\left(\frac{1}{2}\right)^{(\alpha-1) / 2} R^{-1} D_{4} \simeq\left(\frac{1}{2}\right)^{(\alpha-1) / 2}\left(\frac{1}{1+i}\right) D_{4}$.

Thus, in this section, we have the interference alignment onto a lattice for $k<0$. So we can conclude that, by using the binary cyclotomic field $\mathbb{Q}\left(\xi_{8}\right)$, we obtained the construction of a double infinite nested lattice partition chain in dimension 4 (real) or 2 (complex).

\section{CONCLUSIONS}

In this work, in order to realize interference alignment onto a lattice, we explicited one example of channel quantization. This example is related to the dimensions 4 (real) or 2 (complex) and we made use of the binary cyclotomic field $\mathbb{Q}\left(\xi_{8}\right)$, where $\xi_{8}$ is the 8 -th root of unity. So we obtained the construction of a double infinite nested lattice partition chain in dimension 4 (real) or 2 (complex) in order to realize interference alignment onto a lattice.

\section{REFERÊNCIAS}

[1] B. Nazer and M. Gastpar, "Compute-and-forward: Harnessing interference through structured codes," IEEE Trans. Inform. Theory, v. 57, pp. 6463-6486, October 2011.

[2] R. Zamir, "Lattices are everywhere," Proceedings of the 4th Annual Workshop on Information Theory and its Applications (ITA), January 2009.

[3] U. Erez and R. Zamir, "Achieving $\frac{1}{2} \log (1+\mathrm{SNR})$ on the AWGN channel with lattice encoding and decoding," IEEE Trans. Inform. Theory, v. 50, pp. 2293-2314, October 2004.

[4] F. Oggier, Algebraic Methods for Channel Coding. École Polytechnique de Lausanne, Lausanne, 2005.

[5] G. D. Forney, "Coset Codes - Part I: Introduction and Geometrical Classification," IEEE Trans. Inform. Theory, v. 34, pp. 1123-1151, September 1998 . 